\title{
Theoretical and experimental study of the product branching in the reaction of acetic acid with $\mathrm{OH}$-radials
}

\section{- Supporting Information -}

\author{
F. De Smedt, X.V. Bui, T.L. Nguyen, J. Peeters, L. Vereecken*
}

Department of Chemistry, Celestijnenlaan 200F, 3001 Leuven, Belgium

Below the geometries, energies and vibrational wavenumbers of all pertaining species in the $\mathrm{CH} 3 \mathrm{COOH}+\mathrm{OH}$ reaction are listed.

Levels of theory:

$$
\begin{aligned}
& \text { B3LYP-DFT/6-311++G(2df,2pd) } \\
& \text { energy given as E(UB+HF-LYP/6-311++G(2df,2pd) } \\
& \text { MP2/6-311++G(2df,2pd) } \\
& \quad \text { energy given as E(MP2/6-311++G(2df,2pd) }
\end{aligned}
$$

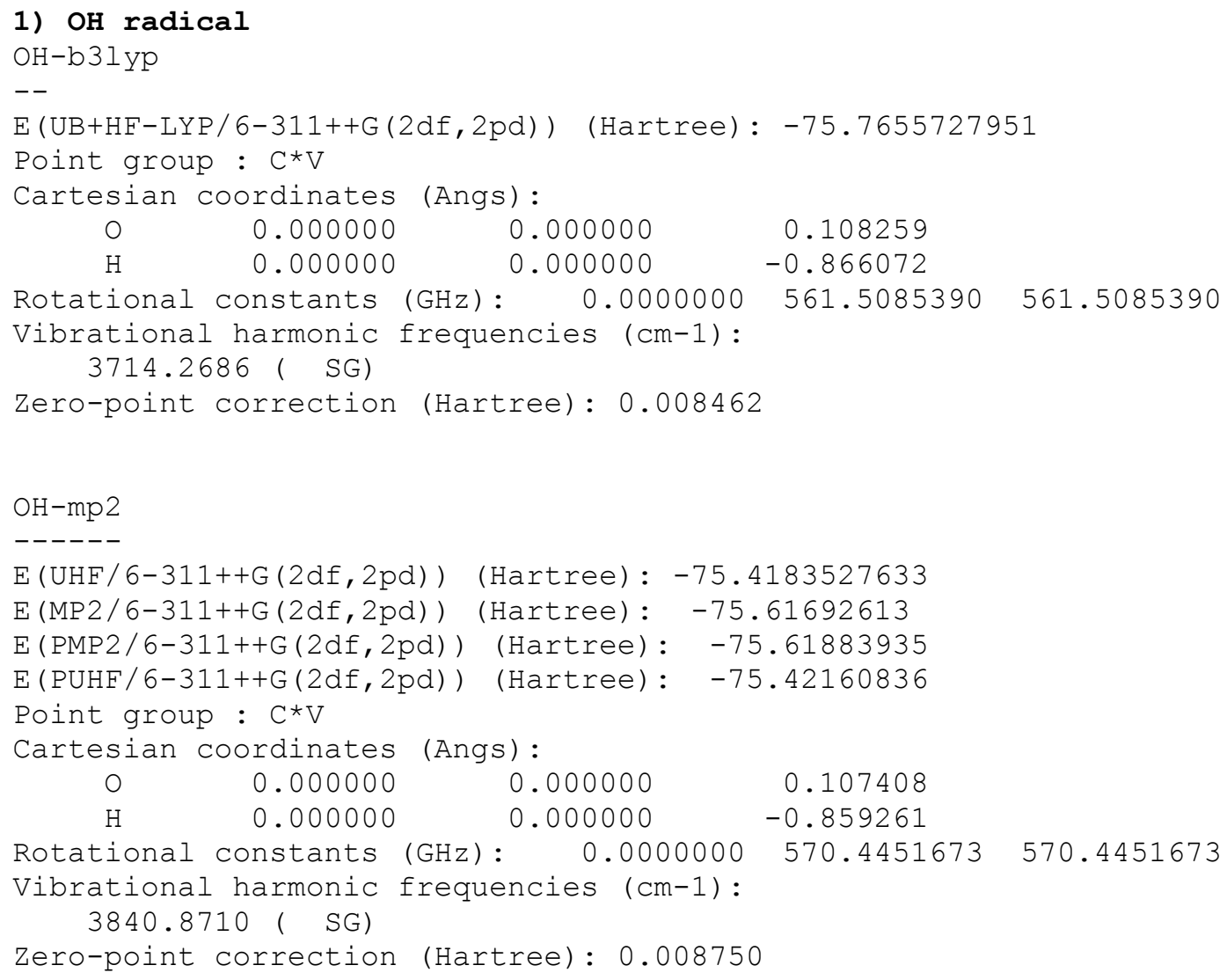

2) $\mathrm{CH}_{3} \mathrm{COOH}$-trans: acetic acid $\mathrm{CH}_{3} \mathrm{COOH}-\mathrm{b} 3 \mathrm{I}_{\text {yp }}$ 


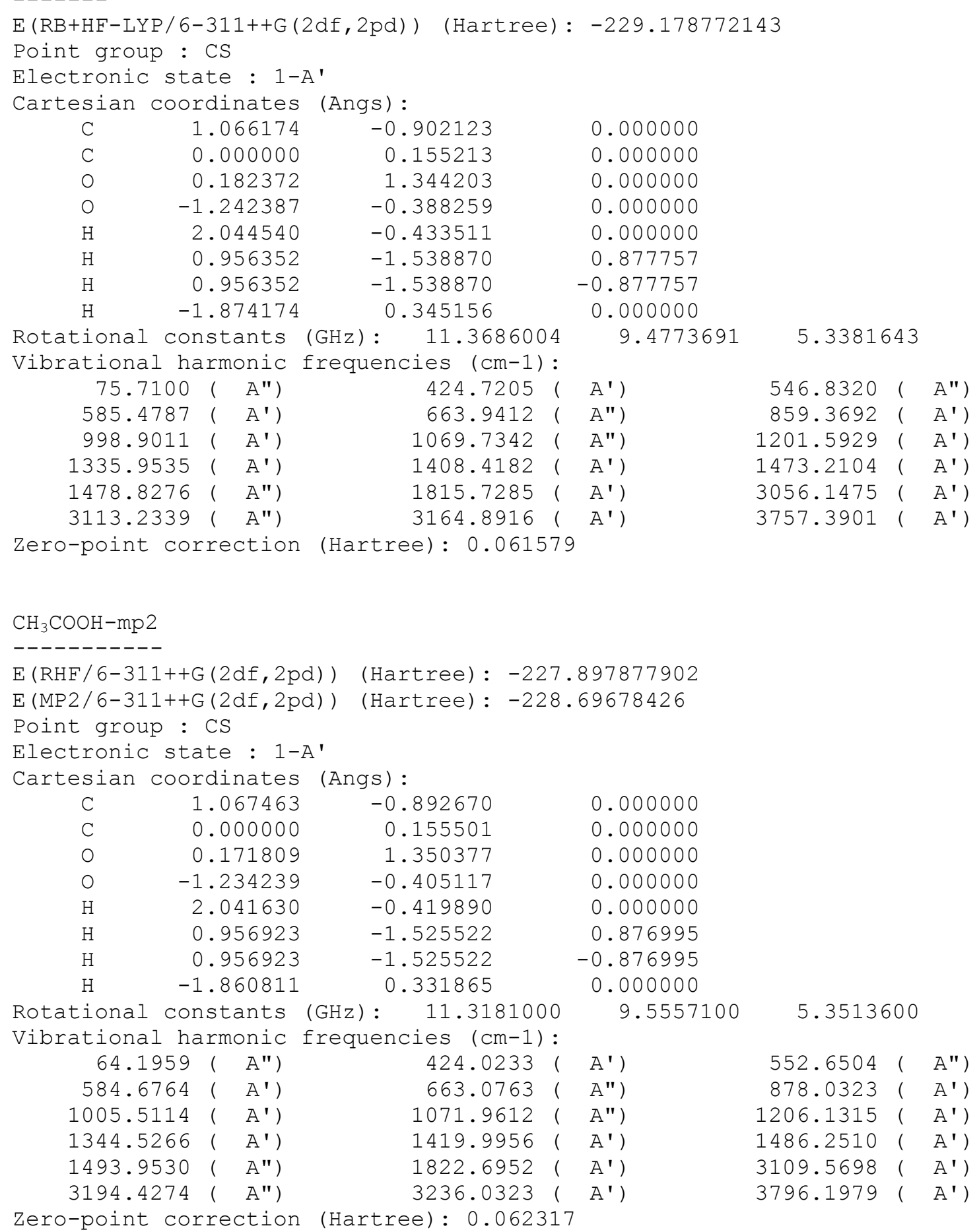

\section{3) $\mathrm{H}_{2} \mathrm{CCOOH}$ radical}

\section{$\mathrm{CH}_{2} \mathrm{COOH}$}

$\mathrm{E}(\mathrm{UB}+\mathrm{HF}-\mathrm{LYP} / 6-311++\mathrm{G}(2 \mathrm{df}, 2 \mathrm{pd}))$ (Hartree) : -228.512595778

Point group : CS

Electronic state : $2-\mathrm{A}^{\prime \prime}$

Cartesian coordinates (Angs):
$\mathrm{C}$
0.000000
0.110269
0.000000 


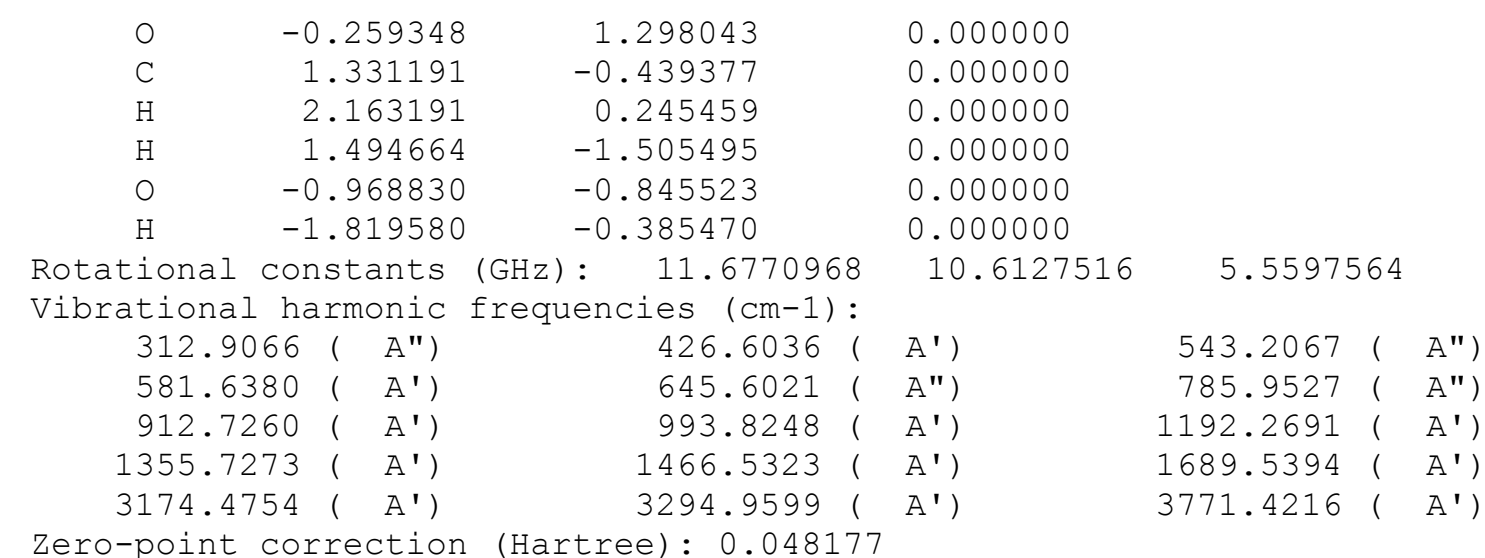

\section{4) $\mathrm{CH}_{3} \mathrm{COO}$ radical}

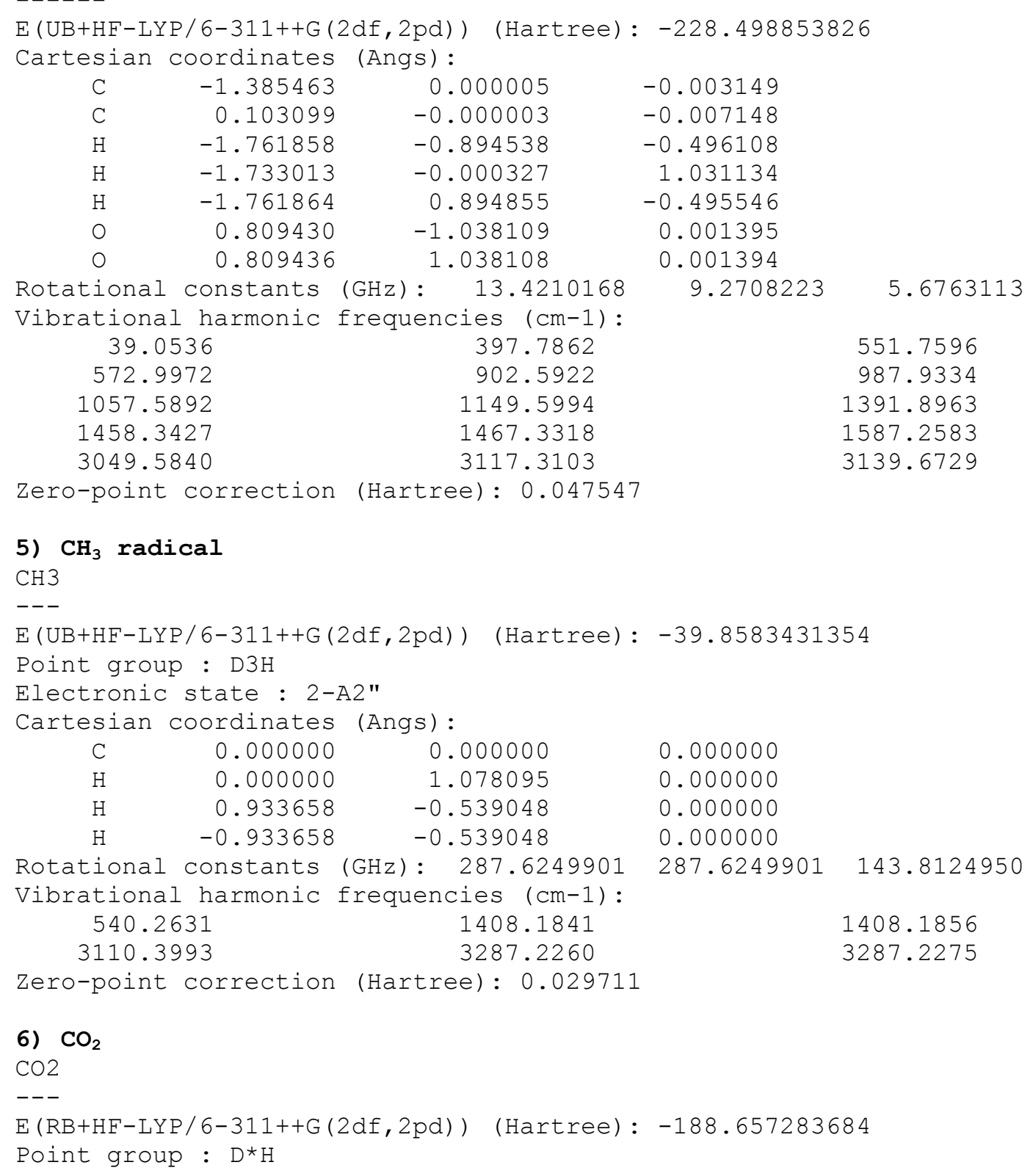

\section{5) $\mathrm{CH}_{3}$ radical}

\section{$\mathrm{CH} 3$}

$\mathrm{E}(\mathrm{UB}+\mathrm{HF}-\mathrm{LYP} / 6-311++\mathrm{G}(2 \mathrm{df}, 2 \mathrm{pd})) \quad$ (Hartree) $:-39.8583431354$

Point group : $\mathrm{D} 3 \mathrm{H}$

Electronic state : 2-A2"

Cartesian coordinates (Angs):

$\begin{array}{rrrr}\mathrm{C} & 0.000000 & 0.000000 & 0.000000 \\ \mathrm{H} & 0.000000 & 1.078095 & 0.000000 \\ \mathrm{H} & 0.933658 & -0.539048 & 0.000000 \\ \mathrm{H} & -0.933658 & -0.539048 & 0.000000\end{array}$

Rotational constants (GHz): $287.6249901 \quad 287.6249901 \quad 143.8124950$

Vibrational harmonic frequencies (cm-1):
540.2631
1408.1841
3287.2260
1408.1856

3110.3993

3287.2275

Zero-point correction (Hartree) : 0.029711

\section{6) $\mathrm{CO}_{2}$}

$\mathrm{CO} 2$

$\mathrm{E}(\mathrm{RB}+\mathrm{HF}-\mathrm{LYP} / 6-311++\mathrm{G}(2 \mathrm{df}, 2 \mathrm{pd}))$ (Hartree) : -188.657283684

Point group : $D^{*} H$ 
Electronic state : $1-\mathrm{SGG}$

Cartesian coordinates (Angs)

$\begin{array}{rrrr}0 & 0.000000 & 0.000000 & 1.159596 \\ \mathrm{C} & 0.000000 & 0.000000 & 0.000000 \\ \mathrm{O} & 0.000000 & 0.000000 & -1.159596\end{array}$

Rotational constants (GHz): $\quad 0.0000000 \quad 11.7487535 \quad 11.7487535$

Vibrational harmonic frequencies (cm-1):

678.6873 ( PIU) 678.6873 ( PIU) 1371.2189 ( SGG)
2408.1463 ( SGU)

Zero-point correction (Hartree) : 0.011702

\section{7) $\mathrm{H}_{2} \mathrm{O}$}

$\mathrm{E}(\mathrm{RB}+\mathrm{HF}-\mathrm{LYP} / 6-311++\mathrm{G}(2 \mathrm{df}, 2 \mathrm{pd}))$ (Hartree) : -76.4635412357

Point group : $\mathrm{C} 2 \mathrm{~V}$

Electronic state : 1-A1

Cartesian coordinates (Angs):

$\begin{array}{rrrr}\mathrm{O} & 0.000000 & 0.000000 & 0.116850 \\ \mathrm{H} & 0.000000 & 0.762884 & -0.467400 \\ \mathrm{H} & 0.000000 & -0.762884 & -0.467400\end{array}$

Rotational constants (GHz): $827.0840650 \quad 430.8092194 \quad 283.2636479$

Vibrational harmonic frequencies (cm-1): $1629.8761 \quad 3817.9431$

Zero-point correction (Hartree) : 0.021343

3920.6906

\section{8) $\mathrm{H}_{2} \mathrm{CO}_{3}$}

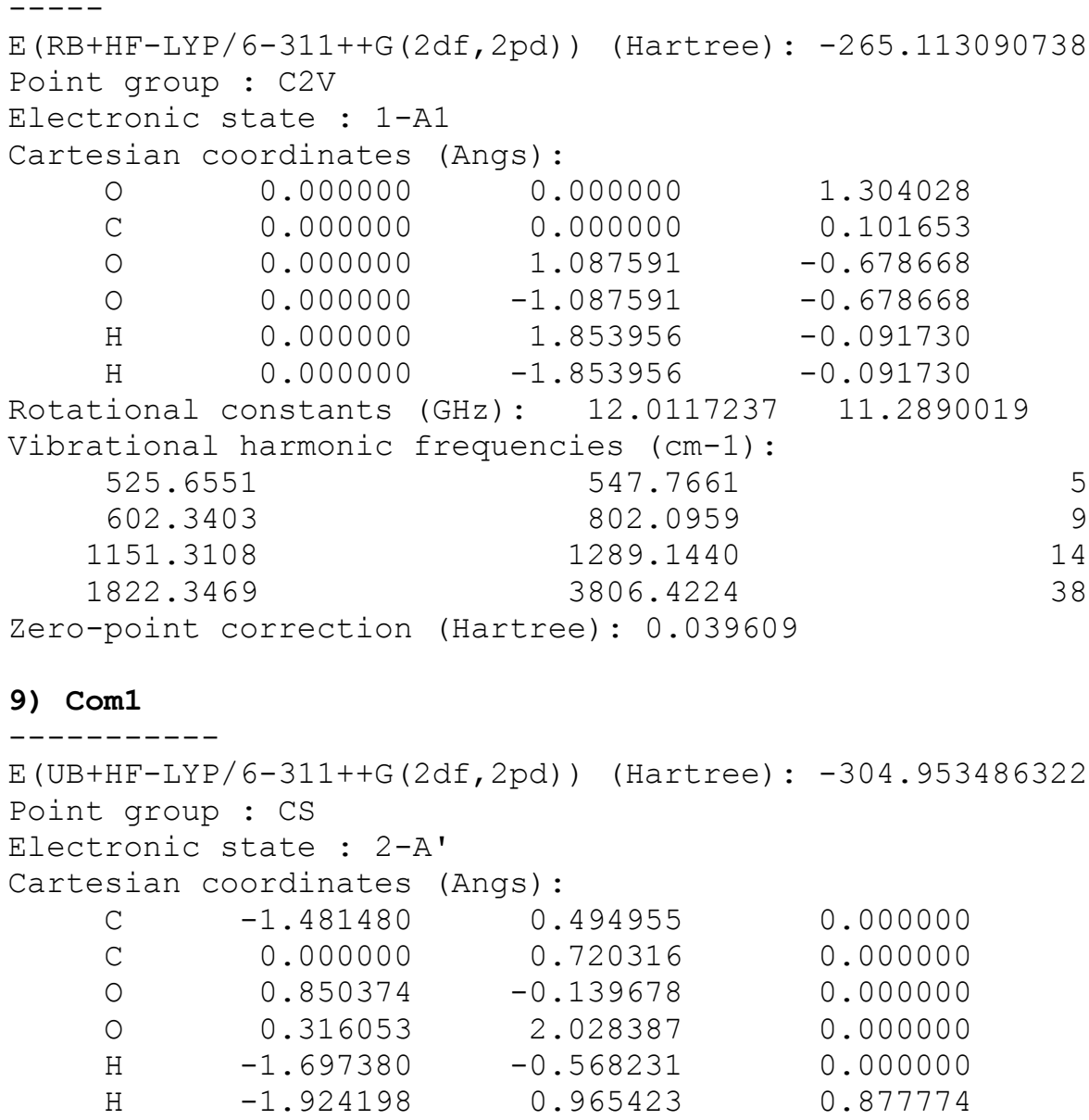




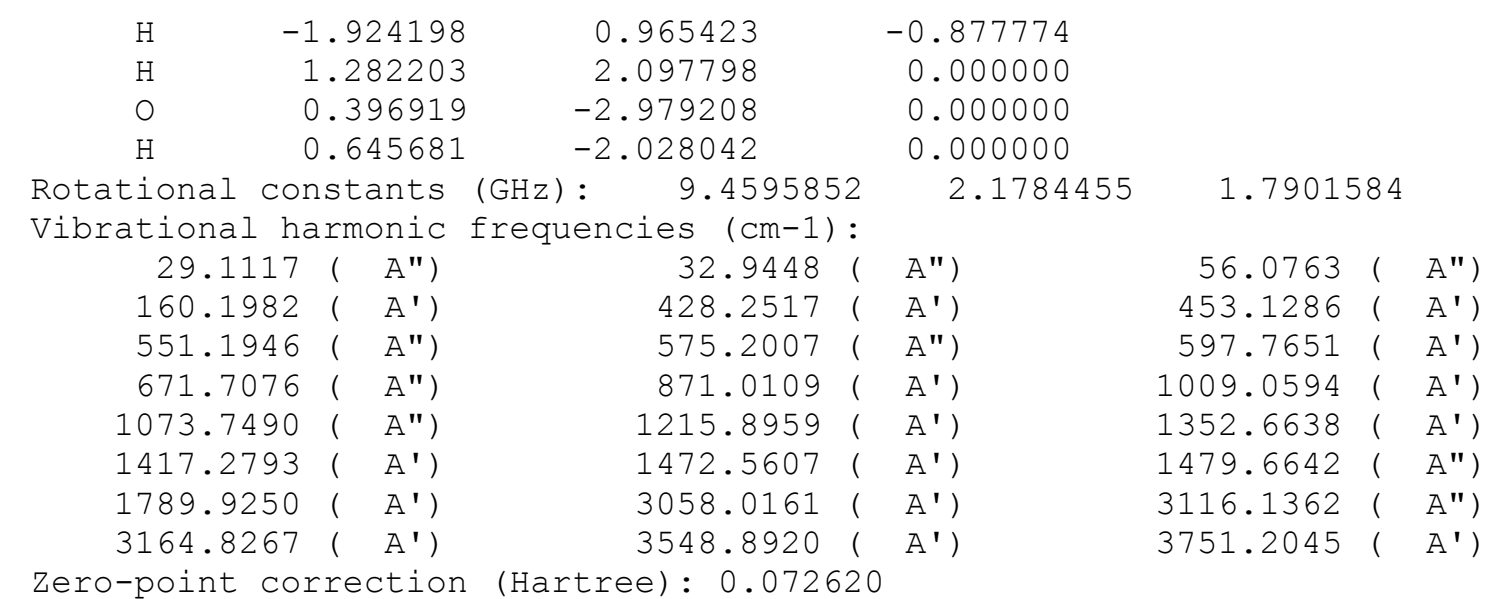

\section{0) Com2}

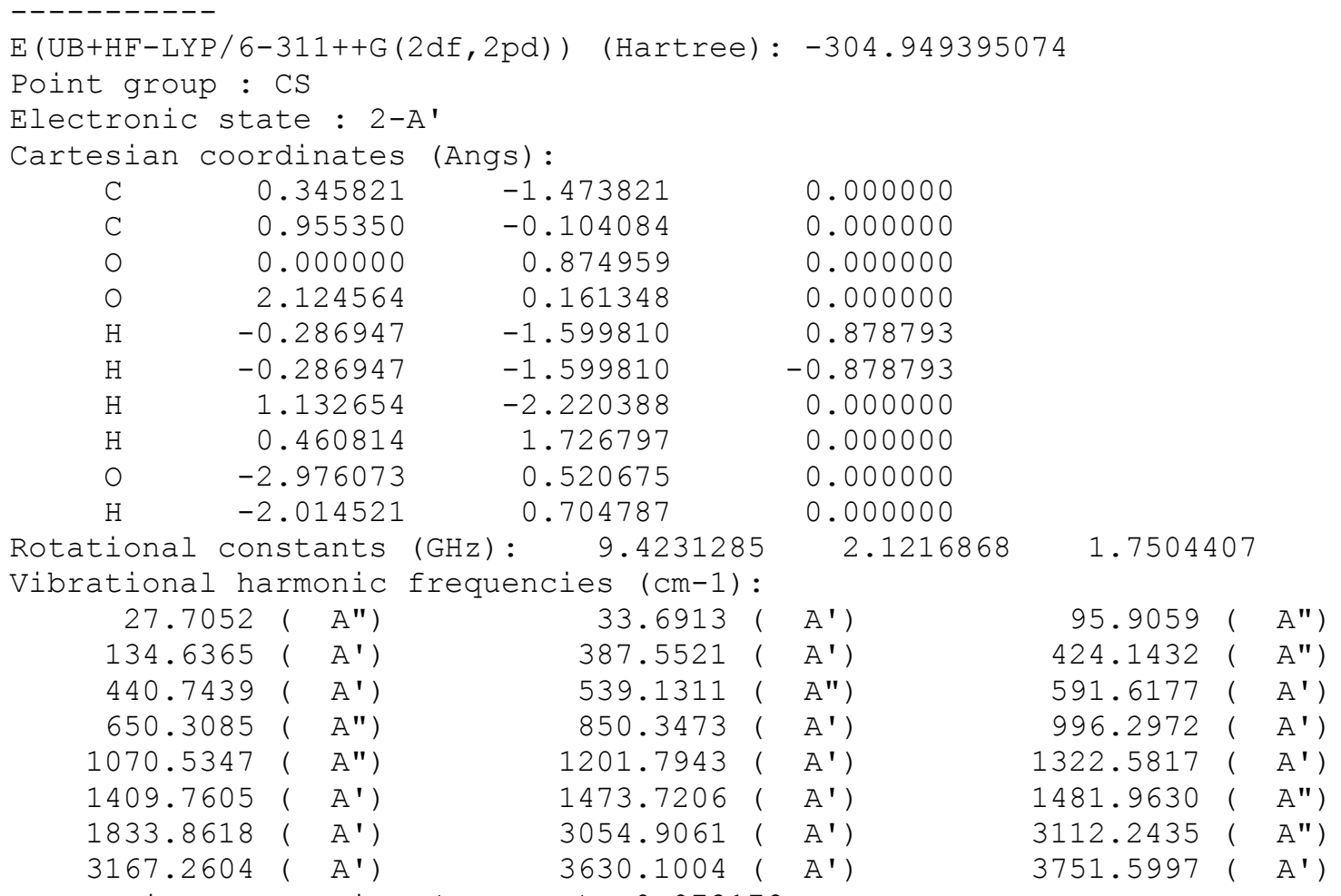

Zero-point correction (Hartree) : 0.072178

\section{1) $\operatorname{Com} 3$}

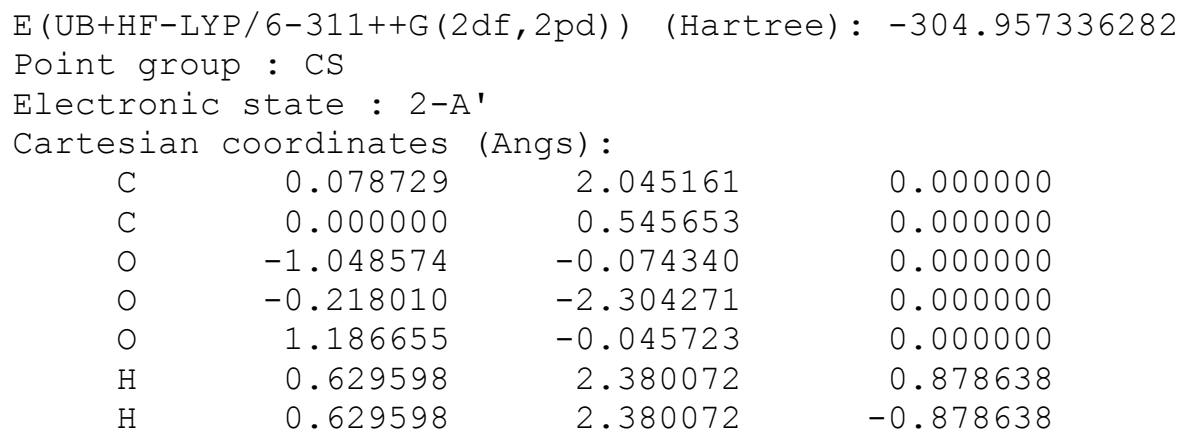




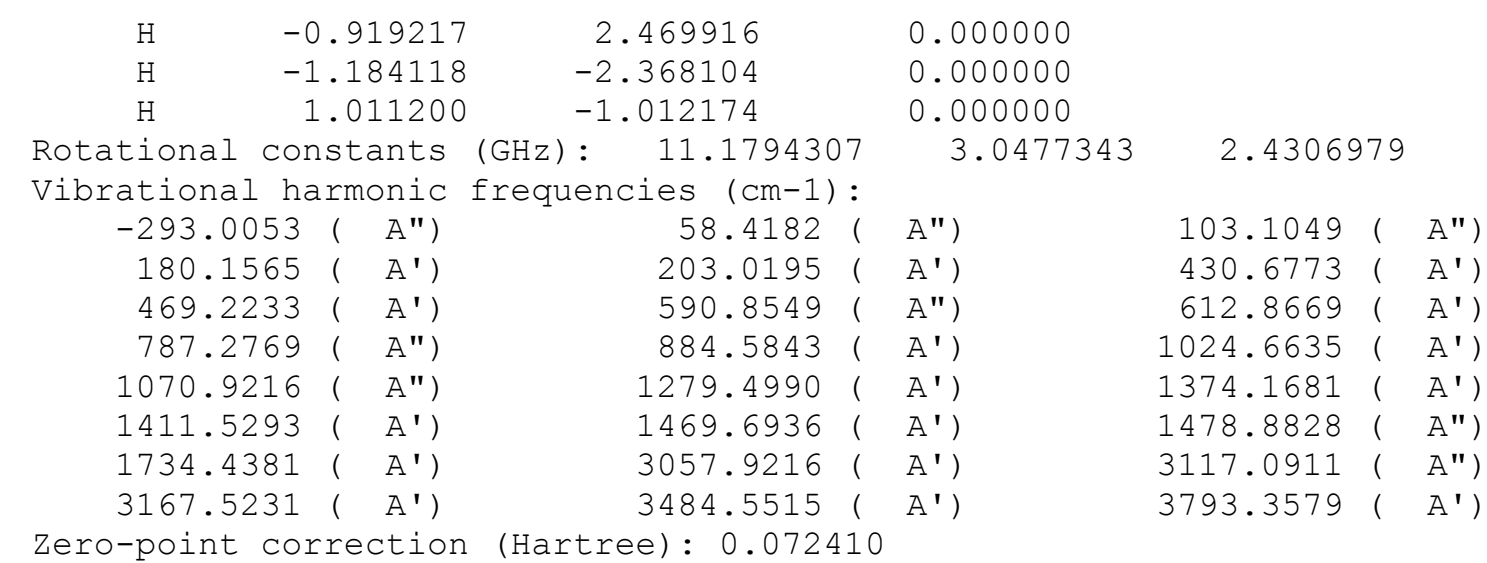

\section{2) $\operatorname{Com} 4$}

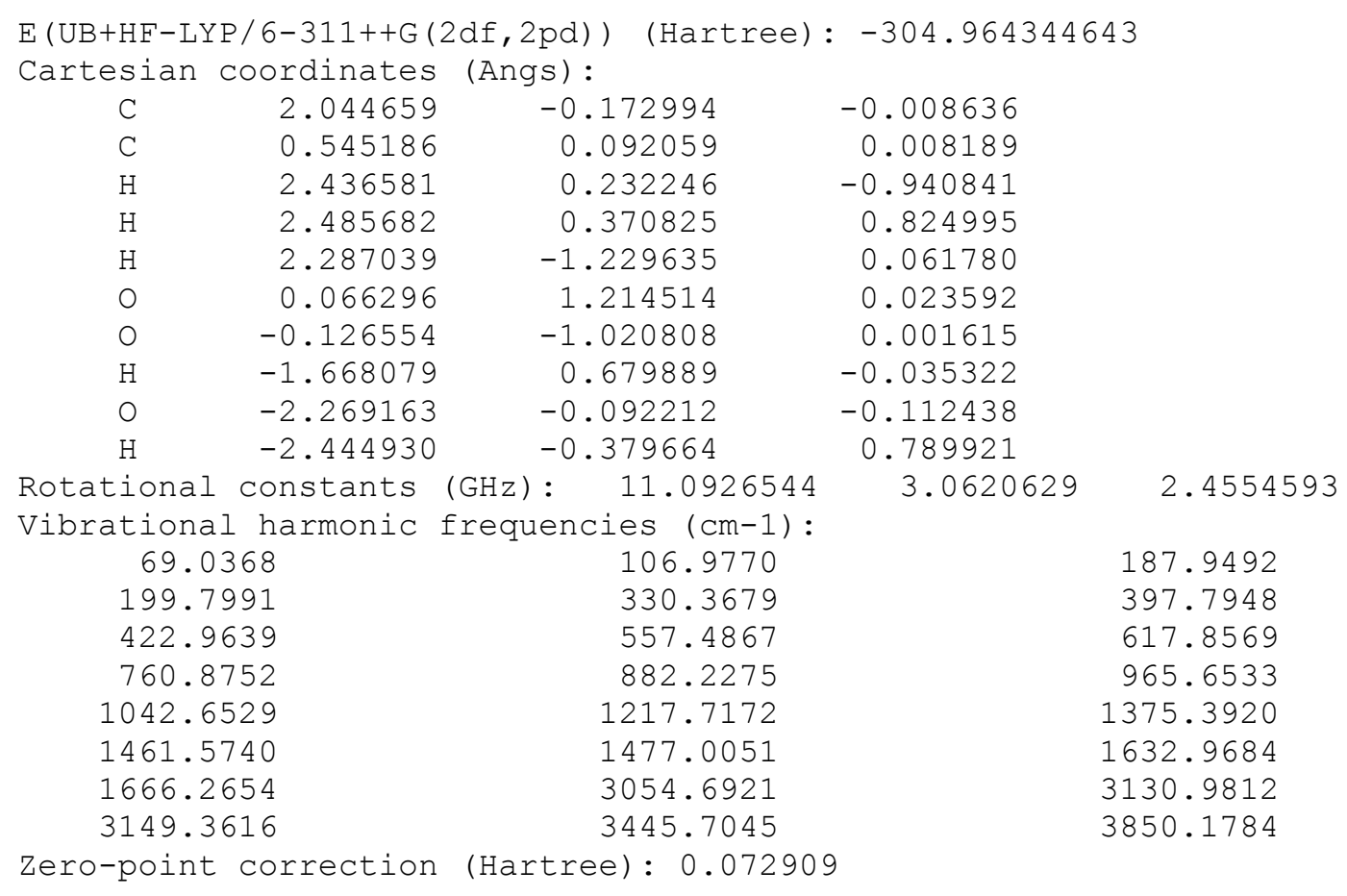

\section{3) Com5}

--

$\mathrm{E}(\mathrm{UB}+\mathrm{HF}-\mathrm{LYP} / 6-311++\mathrm{G}(2 \mathrm{df}, 2 \mathrm{pd}))$ (Hartree) : -304.968182836

Cartesian coordinates (Angs):

$\begin{array}{rrrr}\mathrm{C} & 0.558865 & 1.390646 & 0.152837 \\ \mathrm{C} & 0.832950 & -0.066341 & 0.073379 \\ \mathrm{O} & 1.765652 & -0.579134 & -0.598837 \\ \mathrm{O} & 0.161538 & -0.952515 & 0.658860 \\ \mathrm{H} & -0.468294 & 1.570694 & -0.166534 \\ \mathrm{H} & 0.653904 & 1.716717 & 1.189015 \\ \mathrm{H} & 1.255907 & 1.943554 & -0.473438 \\ \mathrm{H} & -1.919016 & -0.673200 & 0.090425 \\ \mathrm{O} & -2.501069 & 0.022083 & -0.238322 \\ \mathrm{H} & -3.282357 & -0.427075 & -0.570378\end{array}$

Rotational constants (GHz): $\quad 7.3739050 \quad 2.5893901 \quad 2.1790505$

Vibrational harmonic frequencies $(\mathrm{cm}-1)$ : 


$\begin{array}{rrr}21.4069 & 73.5766 & 76.7107 \\ 89.0555 & 126.2563 & 243.5132 \\ 359.8708 & 399.1359 & 561.8774 \\ 568.3765 & 901.6287 & 992.7348 \\ 1063.9877 & 1154.0048 & 1394.2344 \\ 1458.2520 & 1466.7350 & 1587.0922 \\ 1628.3178 & 3046.2865 & 3115.7275 \\ 3137.9989 & 3778.0499 & 3900.7243\end{array}$

\section{4) Com6}

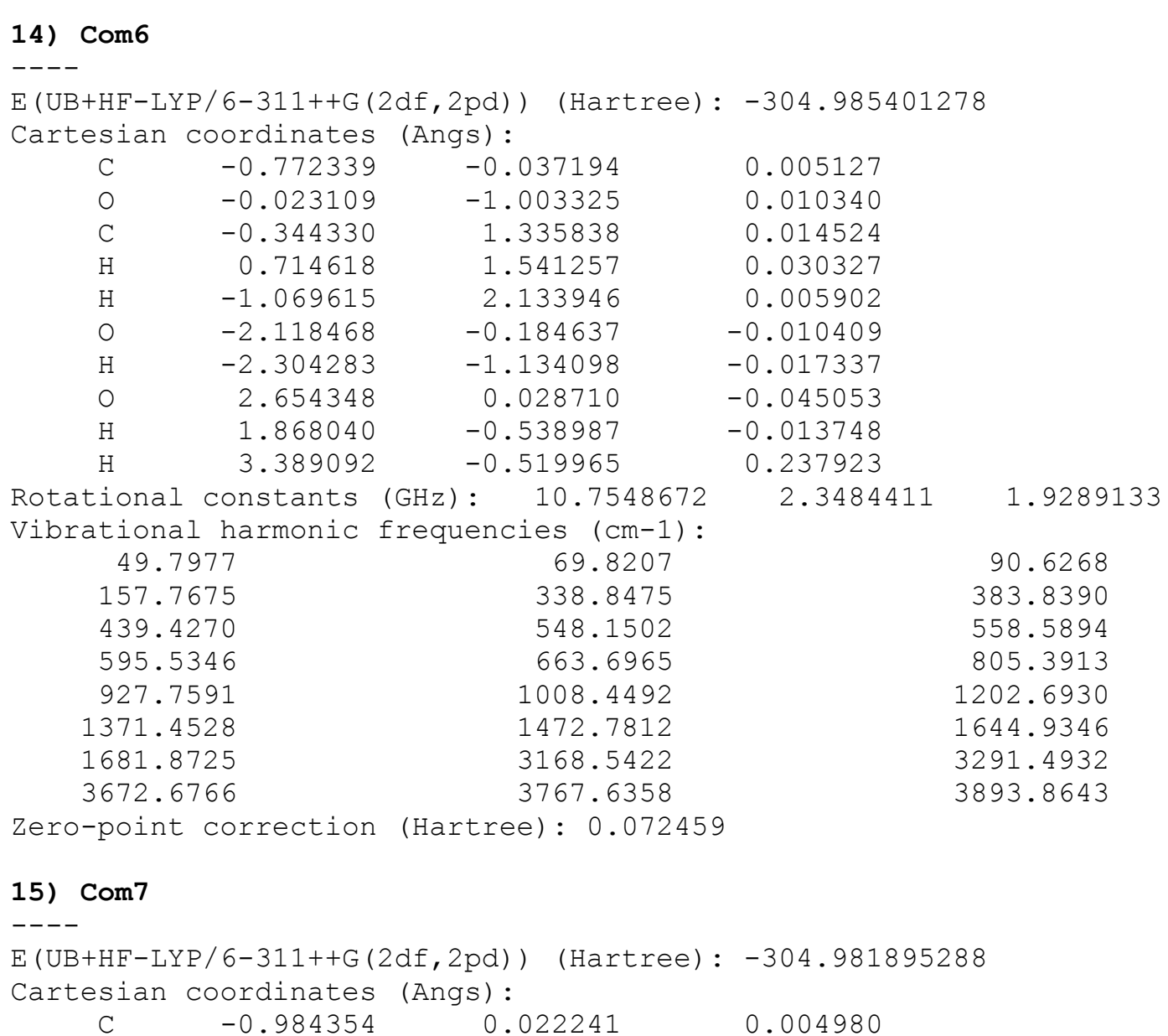

\section{5) $\operatorname{Com} 7$}

$----$

$\mathrm{E}(\mathrm{UB}+\mathrm{HF}-\mathrm{LYP} / 6-311++\mathrm{G}(2 \mathrm{df}, 2 \mathrm{pd}))$ (Hartree) : -304.981895288

Cartesian coordinates (Angs):

$$
\begin{array}{r}
0.004980 \\
0.016016 \\
0.021198 \\
0.036360 \\
0.015021 \\
-0.016870 \\
-0.006258 \\
-0.074982 \\
-0.010557 \\
0.415054
\end{array}
$$

Rotational constants (GHz): 10.3657832

Vibrational harmonic frequencies (cm-1):
40.7765
131.5939
77.6286
437.8226
282.8846
586.2731
443.0970
652.4504
895.5348
1013.3304

1.8557395

106.4727

350.6119

527.5450

801.6241

1196.4020 


$\begin{array}{ccc}1343.1144 & 1474.7827 & 1637.2762 \\ 1694.6152 & 3166.7184 & 3291.0436 \\ 3766.4339 & 3771.3983 & 3900.6985 \\ \text { zero-point correction (Hartree) }: 0.071968 & \end{array}$

\section{6) Com 8}

\begin{tabular}{|c|c|c|c|}
\hline \multicolumn{4}{|c|}{$\begin{array}{l}\text { E (UB+HF-LYP/6-311++G }(2 \mathrm{df}, 2 \mathrm{pd})) \text { (Hartree) : }-304.946846714 \\
\text { Cartesian coordinates (Angs): }\end{array}$} \\
\hline-1.496474 & -0.125657 & -0.064143 & \\
\hline 0.005868 & 0.040844 & 0.045234 & \\
\hline 0.523600 & -0.389095 & 1.243467 & \\
\hline 0.745633 & -0.805668 & -0.846221 & \\
\hline 0.327259 & 1.350573 & -0.252709 & \\
\hline-1.986646 & 0.488224 & 0.689674 & \\
\hline-1.827973 & 0.193870 & -1.050131 & \\
\hline-1.770159 & -1.166179 & 0.100408 & \\
\hline 0.485853 & -1.723097 & -0.695888 & \\
\hline 1.270618 & 1.469585 & -0.086900 & \\
\hline $\begin{array}{l}\text { tational constants } \\
\text { brational harmonic }\end{array}$ & $(\mathrm{GHz}): \quad 5.7570814$ & 5.3840942 & 4.8985534 \\
\hline rational harmonic & frequencies $(\mathrm{cm}-1)$ : & & \\
\hline 213.1430 & 266.0082 & & 334.1363 \\
\hline 374.0017 & 381.1692 & & 468.2761 \\
\hline 535.9728 & 543.1851 & & 801.7244 \\
\hline 849.6425 & 969.3801 & & 1067.1648 \\
\hline 1111.6334 & 1123.2340 & & 1310.7787 \\
\hline 1389.0343 & 1430.1079 & & 1476.1101 \\
\hline 1491.9496 & 3055.7534 & & 3129.7457 \\
\hline 3136.2584 & 3793.8507 & & 3800.4064 \\
\hline
\end{tabular}

Zero-point correction (Hartree) : 0.075300

\section{7) TS1}

TS1-b31yp

\begin{tabular}{|c|c|c|c|}
\hline \multicolumn{4}{|c|}{$\begin{array}{l}\text { E (UB+HF-LYP/6-311++G (2df,2pd)) (Hartree) : }-304.942209928 \\
\text { Cartesian coordinates (Angs): }\end{array}$} \\
\hline 0.379352 & -0.341386 & 0.970854 & \\
\hline-0.759651 & 0.109282 & 0.120469 & \\
\hline-1.079232 & 1.259052 & -0.048884 & \\
\hline-1.394523 & -0.924255 & -0.474887 & \\
\hline 1.349490 & -0.300595 & 0.280338 & \\
\hline 0.573458 & 0.349047 & 1.784011 & \\
\hline 0.326196 & -1.379618 & 1.283835 & \\
\hline-2.093536 & -0.551838 & -1.031994 & \\
\hline 2.450926 & -0.038936 & -0.501404 & \\
\hline 2.308823 & 0.908735 & -0.662724 & \\
\hline ational constants & $(\mathrm{GHz}): \quad 7.2897431$ & 2.6320873 & 2.401704 \\
\hline rational harmonic & frequencies $(\mathrm{cm}-1)$ : & & \\
\hline $\begin{array}{r}-643.9618 \\
163.4132\end{array}$ & $\begin{array}{r}26.7500 \\
343.4605\end{array}$ & & $\begin{array}{r}88.6618 \\
425.5165\end{array}$ \\
\hline 550.7061 & 584.1878 & & 674.8463 \\
\hline 807.2497 & 877.3907 & & 992.1004 \\
\hline 1006.1311 & 1090.3979 & & 1203.1164 \\
\hline 1317.6394 & 1353.5722 & & 1374.8015 \\
\hline 1463.4404 & 1788.8705 & & 3109.5407 \\
\hline
\end{tabular}




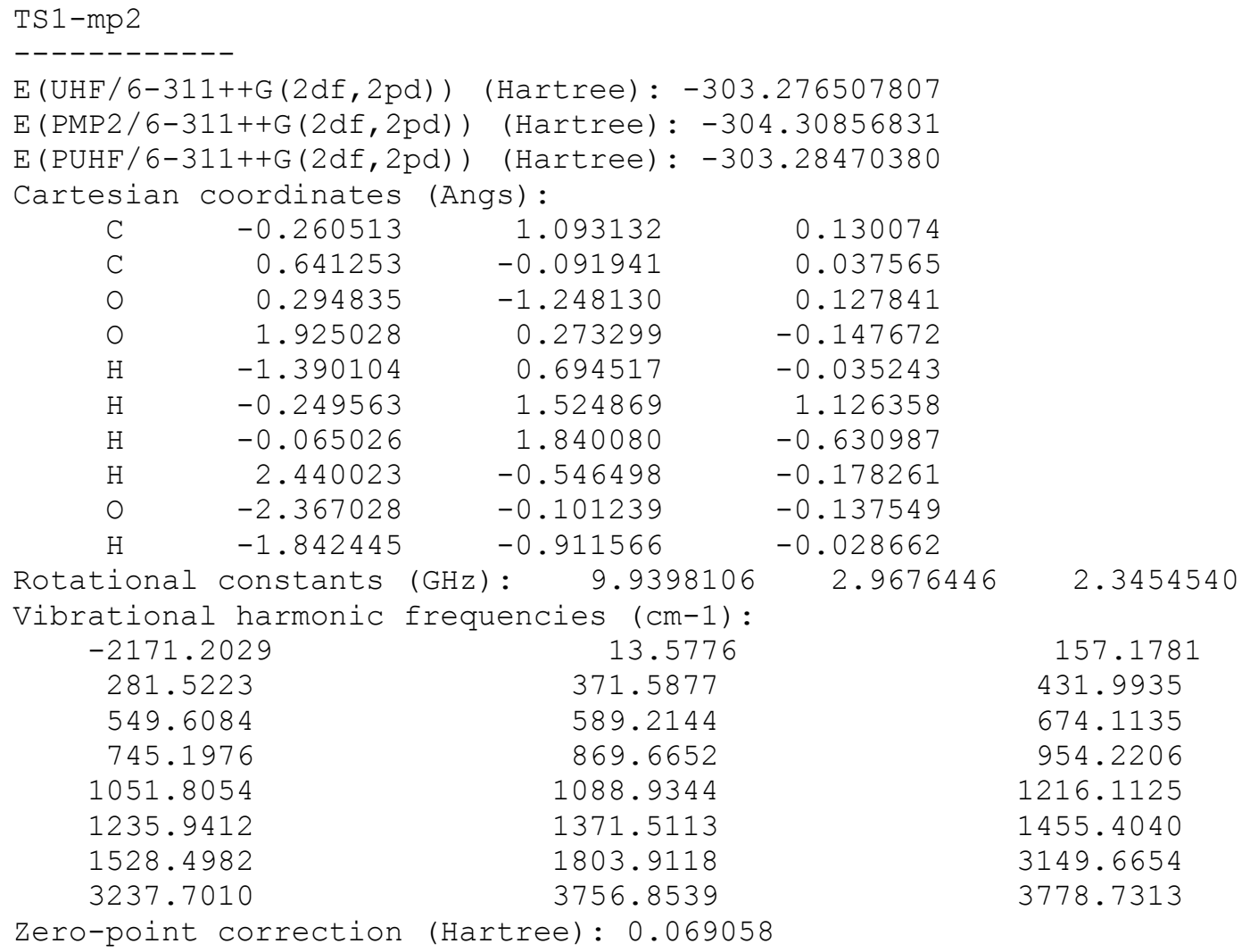

\section{8) TS2}

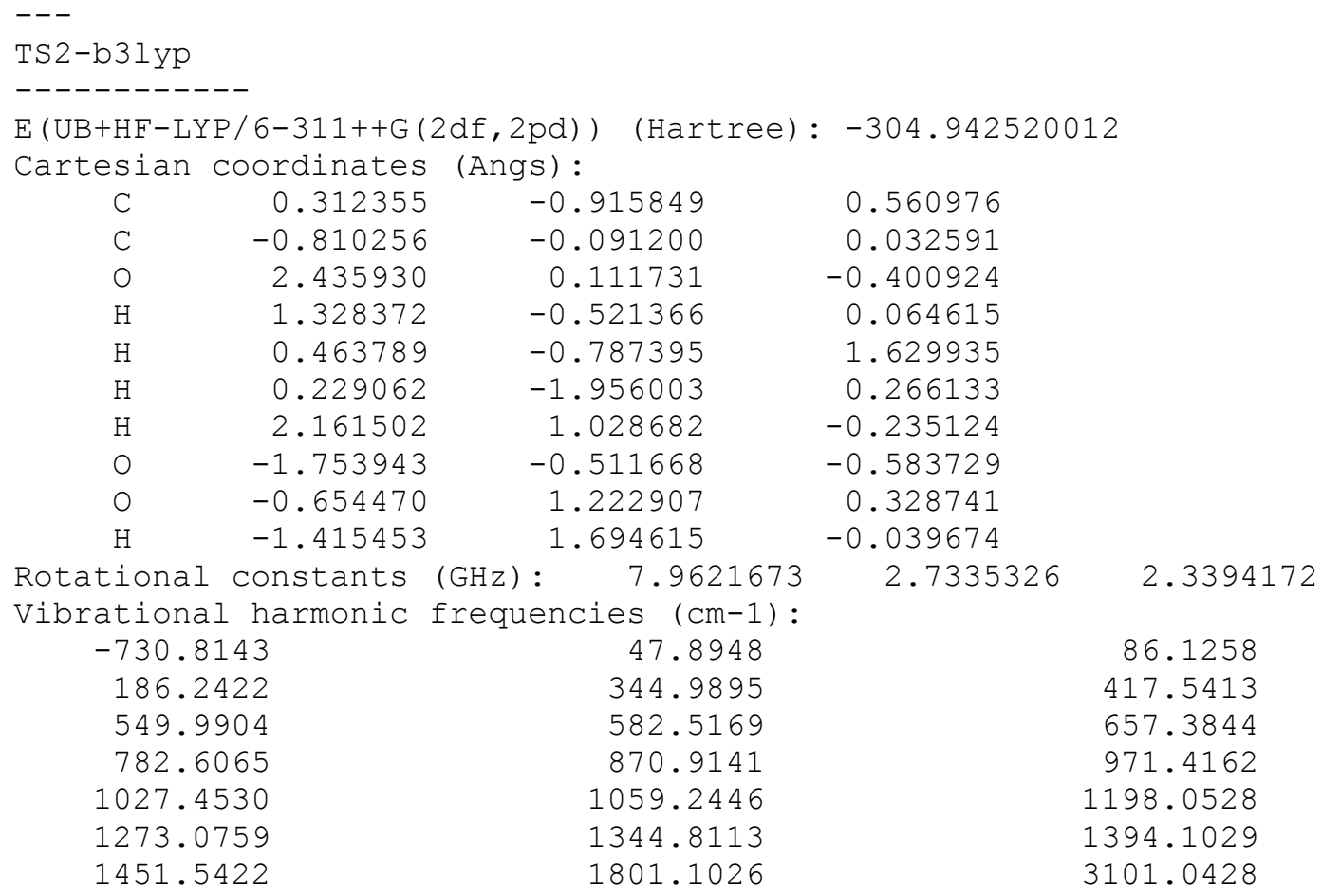




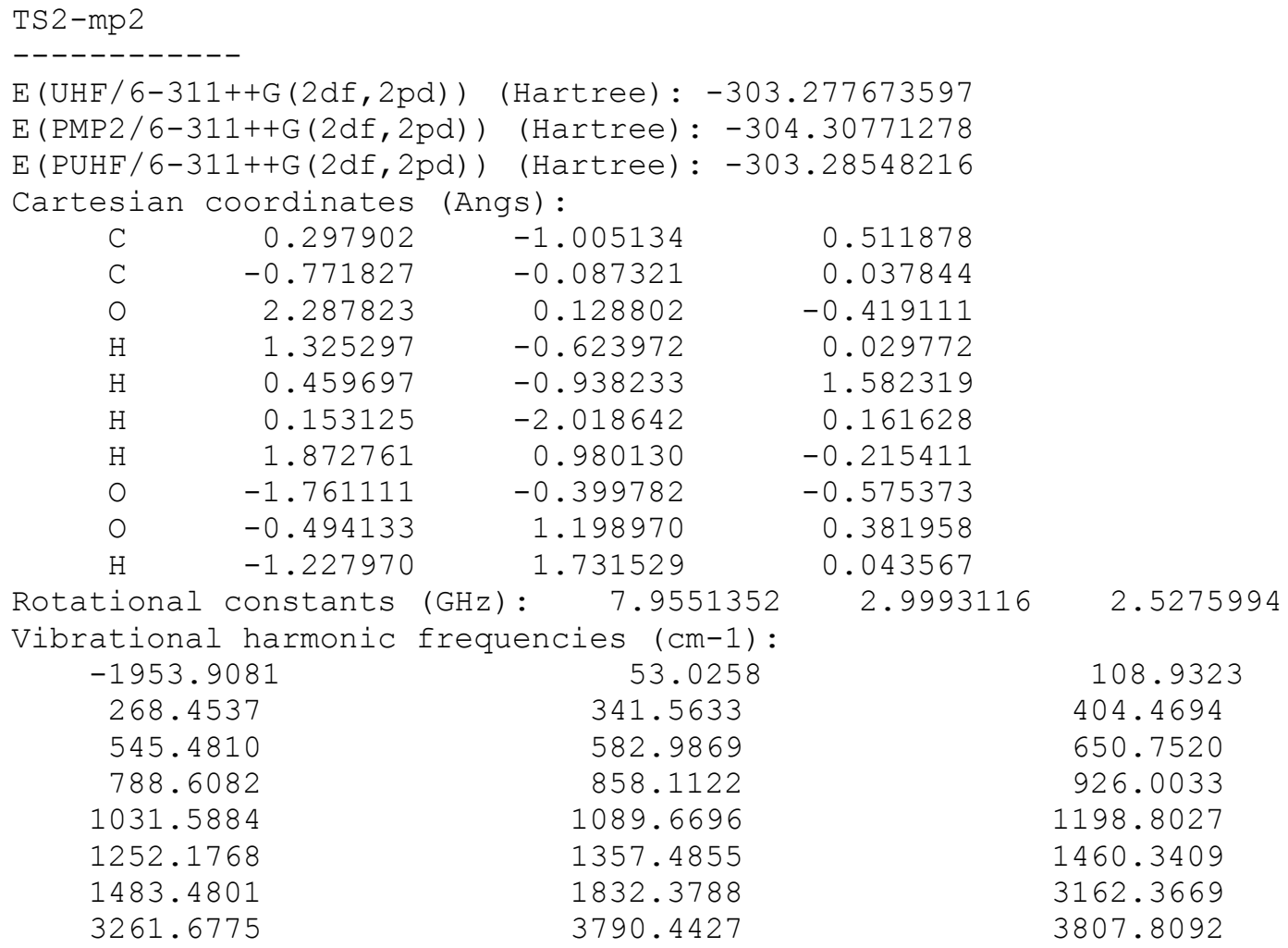

108.9323

404.4694

650.7520

926.0033

1198.8027

1460.3409

3162.3669

3807.8092

\section{9) TS3}

$$
\begin{aligned}
& \text { TS3-b31 yp } \\
& 3.3768401 \quad 2.6739570
\end{aligned}
$$




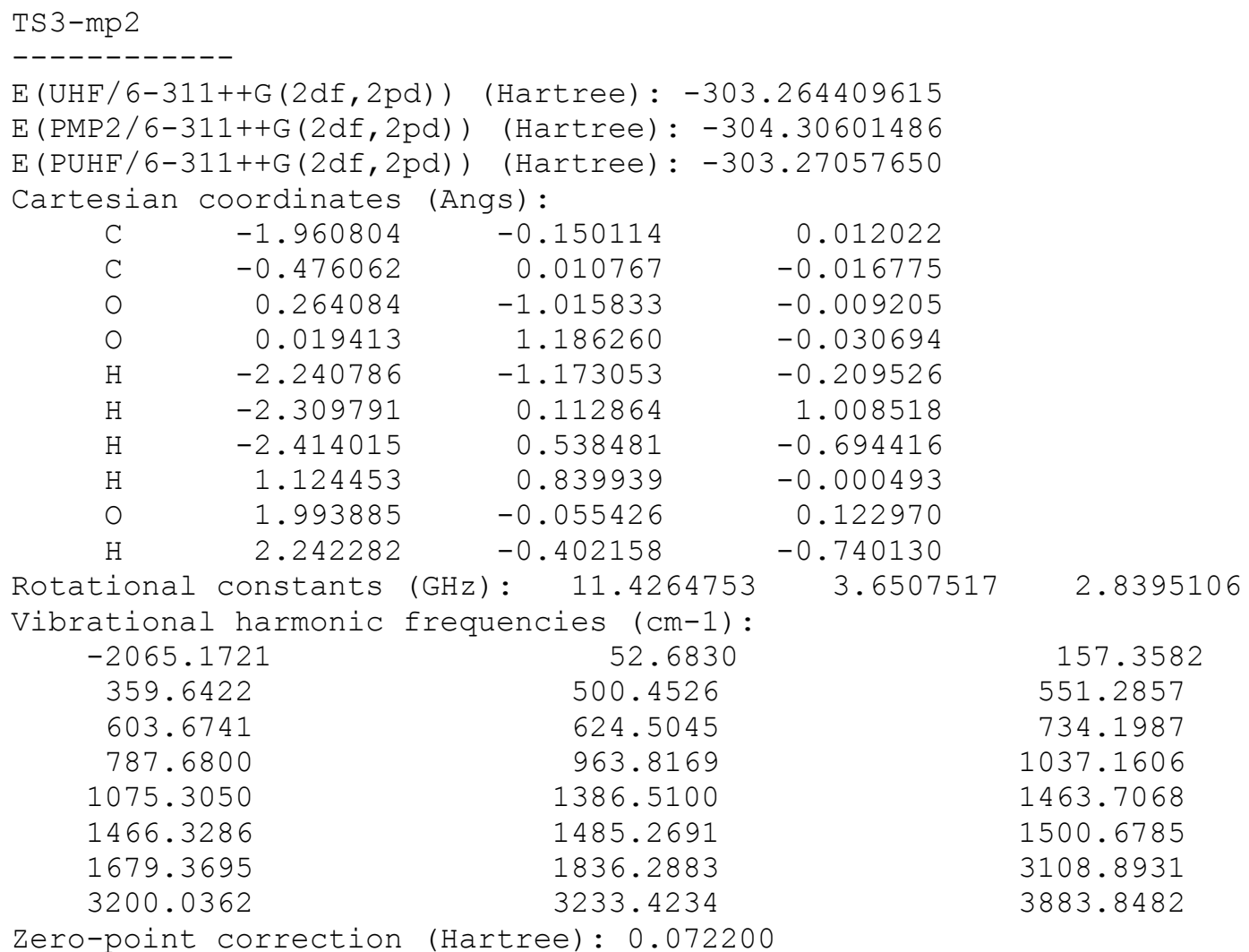

\section{0) TS4}

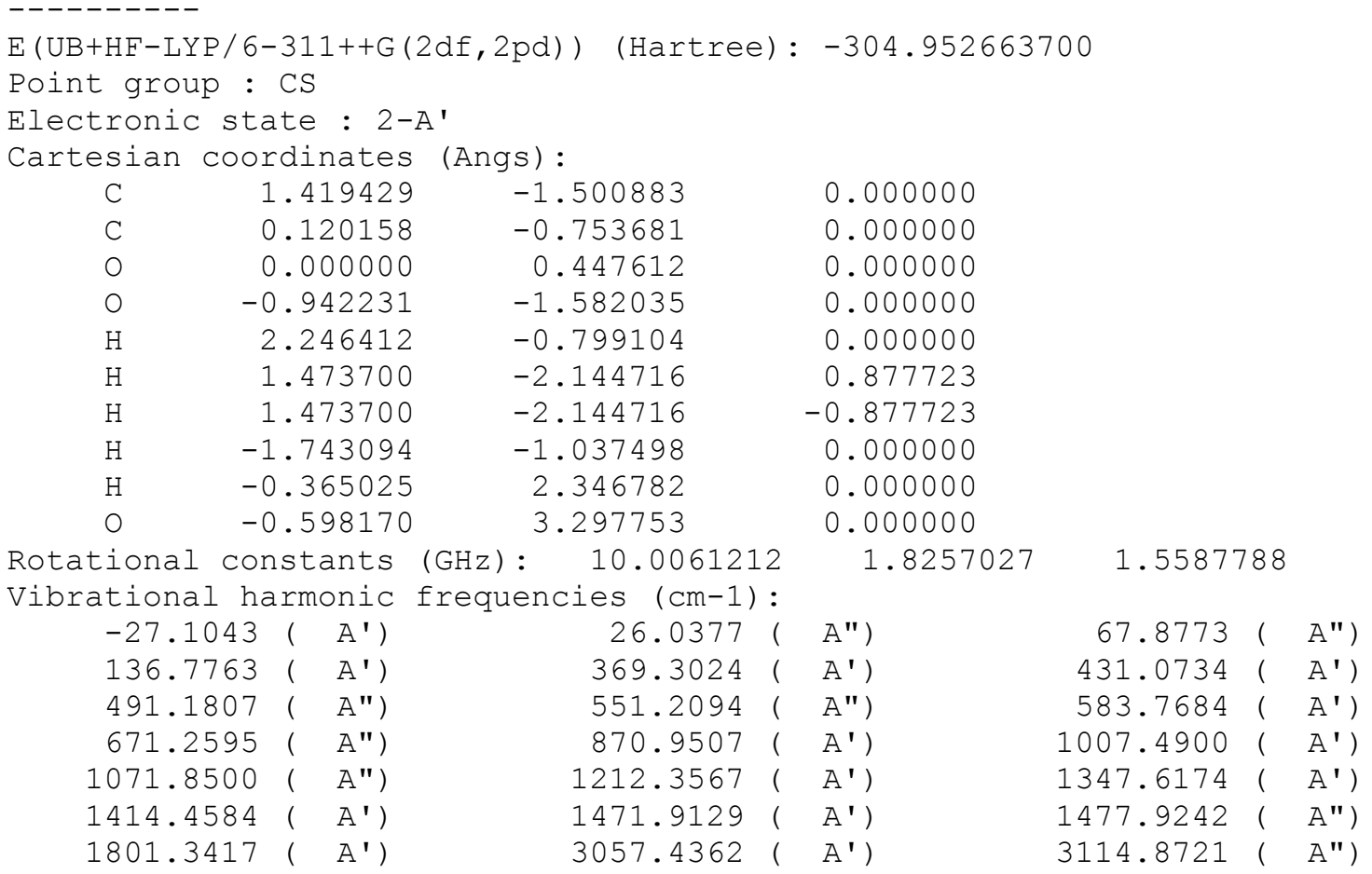


$\begin{array}{crrr}3167.5181\left(\begin{array}{c}\left.A^{\prime}\right) \\ \text { Zero-point correction }\end{array}\right. & 3633.6572(\text { Hartree) }: 0.072285 & \left.A^{\prime}\right) & 3751.5973 \quad\left(\quad A^{\prime}\right)\end{array}$

\section{1) TS5}

$---$

$\mathrm{E}(\mathrm{UB}+\mathrm{HF}-\mathrm{LYP} / 6-311++\mathrm{G}(2 \mathrm{df}, 2 \mathrm{pd}))$ (Hartree) : -304.933736454

Cartesian coordinates (Angs):

C $\quad-1.296581 \quad-0.821595 \quad-0.087485$

C $\quad-0.169474 \quad 0.138685 \quad 0.169401$

$0 \quad 0.531218 \quad 0.105475 \quad 1.223824$

$\begin{array}{llll}0 & 1.271579 & -0.576239 & -0.705269\end{array}$

$\begin{array}{llll}0 & -0.347366 & 1.304785 & -0.480239\end{array}$

$\mathrm{H} \quad-2.181792 \quad-0.458235 \quad 0.436924$

$\mathrm{H}-1.517074 \quad-0.871725 \quad-1.150297$

$\mathrm{H}-1.041554 \quad-1.805388 \quad 0.293911$

$\mathrm{H} \quad 1.543262 \quad-1.349231 \quad-0.186514$

$\mathrm{H} \quad 0.350039 \quad 1.909876 \quad-0.192054$

Rotational constants ( $\mathrm{GHz}): \quad 5.7532656$

Vibrational harmonic frequencies (cm-1):

$315.1250 \quad 351.9519$

$\begin{array}{ll}490.2175 & 545.5824\end{array}$

$845.3744 \quad 867.4506$

$\begin{array}{ll}1030.3958 & 1190.2981\end{array}$

$1408.8191 \quad 1470.7889$

$1520.8619 \quad 3056.5491$

$4.9655267 \quad 4.4254272$

3162.7531

3762.3751

250.0319

436.8265

600.2188

1011.8909

1338.6013

1478.6735

3127.8518

3769.6514

Zero-point correction (Hartree) : 0.073437

\section{2) TS6}

\section{- -}

$\mathrm{E}(\mathrm{UB}+\mathrm{HF}-\mathrm{LYP} / 6-311++\mathrm{G}(2 \mathrm{df}, 2 \mathrm{pd})) \quad$ (Hartree) : -304.939036835

Cartesian coordinates (Angs):

$\begin{array}{llll}\mathrm{C} & -1.578399 & -0.000005 & -0.355089\end{array}$

$\begin{array}{llll}\mathrm{C} & 0.237324 & 0.000000 & 0.128285\end{array}$

$\begin{array}{lllr}0 & 0.065395 & -0.000004 & 1.373397\end{array}$

$0 \quad 0.731302 \quad-1.105701 \quad-0.529150$

$0 \quad 0.731294 \quad 1.105710 \quad-0.529144$

$\mathrm{H} \quad-1.992771 \quad 0.907970 \quad 0.060904$

$\mathrm{H} \quad-1.451983 \quad-0.000006 \quad-1.430217$

$\mathrm{H} \quad-1.992768 \quad-0.907981 \quad 0.060905$

$\mathrm{H} \quad 0.630035 \quad-1.850446 \quad 0.074203$

$\mathrm{H} \quad 0.630003 \quad 1.850454 \quad 0.074206$

$\begin{array}{lllll}\text { Rotational constants (GHz): } & 5.5948917 & 4.9861811 & 4.7509123\end{array}$

Vibrational harmonic frequencies (cm-1):

$\begin{array}{ll}-509.6827 & 195.1144\end{array}$

$283.3661 \quad 355.0951$

$535.9514 \quad 557.7881$

$\begin{array}{ll}780.6337 & 797.3102\end{array}$

$1068.5208 \quad 1159.1164$

$1370.0177 \quad 1426.3930$

$1484.0018 \quad 3085.5166$

$3234.8827 \quad 3821.8911$

Zero-point correction (Hartree): 0.073049

276.9682

423.5051

592.4807

872.2594

1242.0867

1447.7832

3230.9124

3823.1498

\section{3) TS7}


$\mathrm{E}(\mathrm{UB}+\mathrm{HF}-\mathrm{LYP} / 6-311++\mathrm{G}(2 \mathrm{df}, 2 \mathrm{pd}))$ (Hartree) : -228.490151991

Cartesian coordinates (Angs):

$\begin{array}{rrrr}\mathrm{C} & 1.290545 & -0.341242 & -0.000076 \\ \mathrm{C} & -0.232970 & -0.013385 & -0.000128 \\ \mathrm{H} & 1.937390 & 0.530743 & -0.001710 \\ \mathrm{H} & 1.459799 & -0.934526 & 0.896749 \\ \mathrm{H} & 1.459169 & -0.938391 & -0.894252 \\ \mathrm{O} & -0.303124 & 1.280847 & -0.000001 \\ \mathrm{O} & -1.097102 & -0.847106 & 0.000056\end{array}$

Rotational constants (GHz): 11.9287708

Vibrational harmonic frequencies (cm-1):

$\begin{array}{rr}-435.0909 & 102.8121 \\ 602.7143 & 651.6764 \\ 998.6624 & 1114.3696 \\ 1435.2263 & 1475.5670 \\ 3052.4310 & 3145.7754\end{array}$

$9.8998466 \quad 5.6039418$

-point correction (Hartree) : 0.045941

24) TS8

$---$

Cartesian coordinates (Angs):

$\begin{array}{rrrr}\mathrm{C} & 0.375210 & 1.391628 & 0.153542 \\ \mathrm{C} & 0.955667 & -0.340115 & 0.019708 \\ \mathrm{O} & 2.107883 & -0.241417 & -0.318159 \\ \mathrm{O} & 0.054283 & -1.079530 & 0.313261 \\ \mathrm{H} & -0.602236 & 1.330961 & -0.309459 \\ \mathrm{H} & 0.359831 & 1.496240 & 1.231922 \\ \mathrm{H} & 1.109206 & 2.002415 & -0.350234 \\ \mathrm{H} & -1.933334 & -0.601547 & 0.065735 \\ \mathrm{O} & -2.608930 & 0.057428 & -0.139855 \\ \mathrm{H} & -3.344624 & -0.428994 & -0.519452\end{array}$

Rotational constants (GHz): 8.6563410

Vibrational harmonic frequencies ( $\mathrm{cm}-1)$ :

$-287.2660$

110.0661

282.0559

590.0821

845.7992

1429.8356

1882.6327

3249.0733

Zero-point correction (Hartree): 0.069268
28.2992

127.7842

300.4907

619.5310

1167.8012

1448.1598

3081.4896

3759.5791
396.4847

929.4091

1353.1345

1752.9891

3154.4500
2.3497763
1.9296678
90.0784
142.6340
442.7614
780.7728
1273.3683
1634.6642
3220.2679
3898.0338

\section{5) $\mathrm{CH}_{3} \mathrm{COOH}$-cis: acetic acid}

$$
\begin{aligned}
& \mathrm{E}(\mathrm{RB}+\mathrm{HF}-\mathrm{LYP} / 6-311++\mathrm{G}(2 \mathrm{df}, 2 \mathrm{pd})) \quad \text { (Hartree) : }-229.170389830 \\
& \text { Cartesian coordinates (Angs): } \\
& \begin{array}{rr}
\mathrm{C} & 1.369879 \\
\mathrm{C} & -0.129118 \\
\mathrm{O} & -0.680140 \\
\mathrm{O} & -0.866294 \\
\mathrm{H} & 1.681143 \\
\mathrm{H} & 1.860895 \\
\mathrm{H} & 1.681146 \\
\mathrm{H} & -0.296272
\end{array} \\
& -0.055465 \quad 0.00000 \\
& 0.141250 \quad 0.00000 \\
& 1.202625 \quad 0.000000 \\
& -1.004116 \quad 0.00000 \\
& -0.617187 \quad-0.8826 \\
& 0.912115 \quad 0.0000 \\
& \begin{array}{ll}
-0.617008 & 0.8826
\end{array} \\
& \begin{array}{ll}
-1.780694 & 0.0000
\end{array}
\end{aligned}
$$




$\begin{array}{ccr}\text { Vibrational harmonic frequencies }(\mathrm{cm}-1): & \\ 95.3440 & 432.1062 & 468.5731 \\ 598.7660 & 600.8191 & 856.3657 \\ 988.3597 & 1064.5600 & 1201.0415 \\ 1291.7341 & 1399.6256 & 1473.8420 \\ 1488.7902 & 1850.8436 & 3039.7100 \\ 3096.0586 & 3158.2846 & 3814.6444 \\ \text { Zero-point correction (Hartree) }: 0.061327 & \end{array}$

\section{6) TS9}

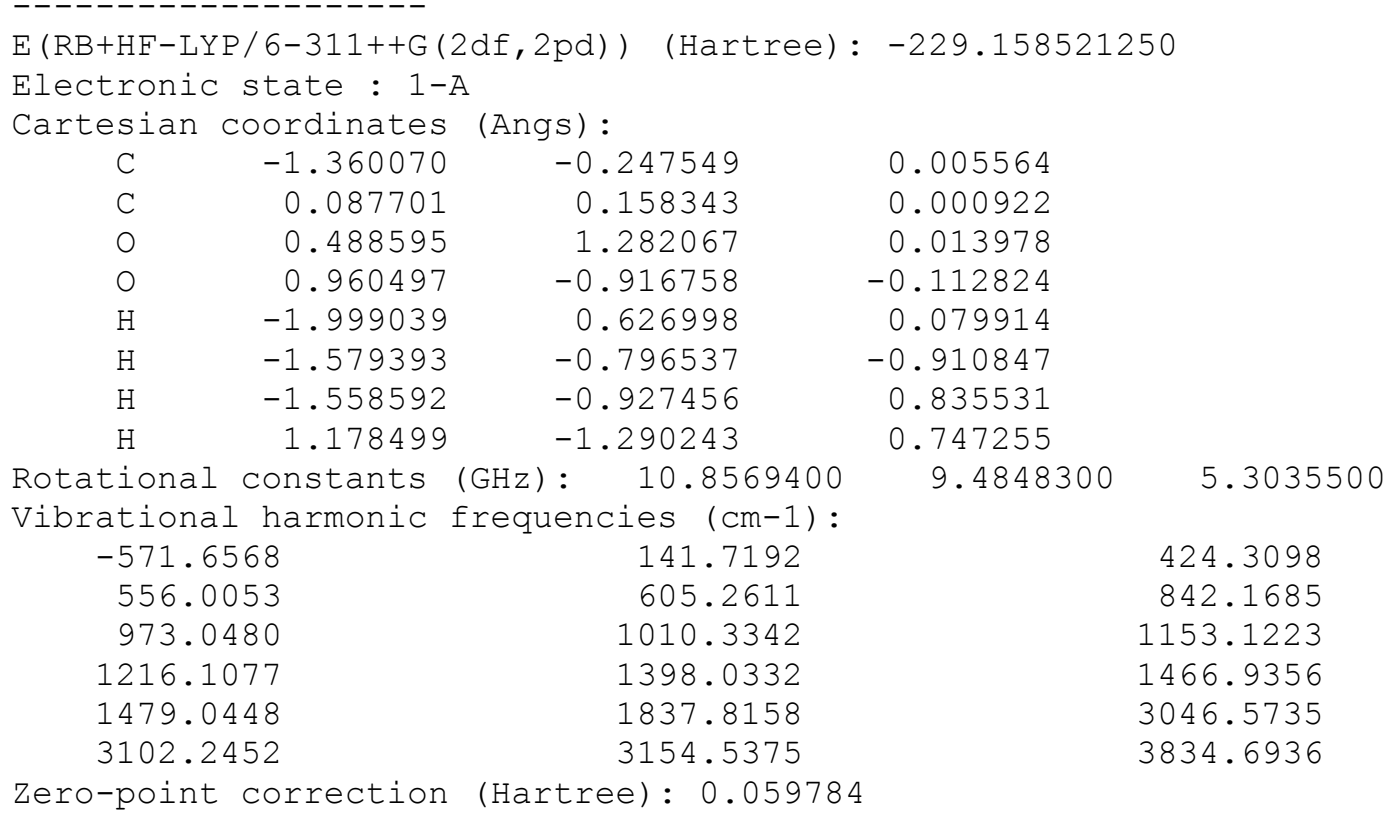

